\title{
Homogénéisation de matériaux périodiques à l'aide de la méthode des éléments finis, dans la limite des grandes longueurs d'onde
}

\author{
Ph. LANGLET, A.-C. HLADKY-HENNION, R. BOSSUT et J.-N. DECARPIGNY
}

I.E.M.N., UMR 9929 du CNRS, Département ISEN, 41 Boulevard Vauban, 59046 Lille cedex, France

\begin{abstract}
Résumé: Un modèle numérique a été développé pour étudier la propagation d'ondes planes harmoniques dans un matériau périodique dans une, deux ou trois directions de l'espace. Les propriétés homogénéisées de matériaux poreux sont déterminées sur un modele anisotrope, dans la limite des grandes longueurs d'onde. Une validation a été faite sur des plaques perforées périodiquement par la mesure des fréquences de résonance.
\end{abstract}

Abstract: A numerical model has been developed to study the propagation of plane harmonic waves in single, doubly or triply periodic materials. The homogenized properties of porous materials are determined with the help of an anisotropic model, in the limit of large wavelengths. A validation has been carried out with periodically perforated plates, the resonance frequencies of which have been measured.

\section{INTRODUCTION.}

La propagation d'une onde acoustique plane et harmonique dans un matériau comportant des cavités ou des inclusions rangées périodiquement dans une, deux ou trois directions de l'espace est susceptible de nombreuses applications, notamment dans les domaines de l'acoustique sous-marine, du traitement des signaux et de l'acoustique médicale. De tels matériaux sont utilisés, par exemple, comme revêtements anéchoïques de structures immergées, comme lignes à retard ou filtres acoustiques. De même, les matériaux piézocomposites interviennent dans la conception de nouveaux transducteurs ultrasonores. Des modèles analytiques exacts, semi-analytiques ou numériques ont été développés pour modéliser le comportement de tels matériaux [1-3], mais sont souvent réduits à des cas particuliers. Le modèle numérique semble le mieux adapté pour simuler le comportement de ces matériaux, sans restriction sur la gémétrie, sur les propriétés des matériaux, ou sur l'angle d'incidence de l'onde acoustique. La méthode des éléments finis, avec l'aide du cođe ATILA, a été utilisée pour étudier la propagation des ondes acoustiques dans des matériaux périodiques [5] et constitue la base de l'outil de simulation mis en oeuvre dans cette communication.

Après un rappel succinct du modèle mathématique, les propriétés homogénéisées dans la limite des grandes longueurs d'onde, de matériaux poreux sont alors recherchées. L'étude expérimentale des fréquences de résonance d'une plaque perforée périodiquement permet de valider l'ensemble des résultats obtenus. 


\section{FORMULATION THEORIQUE.}

Un matériau périodique suivant trois directions de l'espace est entièrement décrit par un réseau défini par trois vecteurs et une cellule élémentaire. Le théorème de Bloch-Floquet permet de réduire la modélisation du matériau à cette cellule élémentaire, qui fait seule l'objet đu maillage éléments finis. Le vecteur d'onde étant fixé, les relations de phase entre les points distants d'un nombre entier de fois le pas du réseau sont déterminées et appliquées sur les points des faces délimitant la cellule élémentaire. La résolution du système se fait par recherche de valeurs propres et vecteurs propres dans une matrice hermitienne, qui sont les pulsations des modes de propagation et leur vecteur des valeurs nodales du champ de déplacement associé. Les courbes de dispersion sont obtenues en faisant varier le nombre d'onde $\mathbf{k}$, norme du vecteur d'onde $\underset{\sim}{\mathrm{k}}$. Elles permettent alors de déterminer les modes de propagation, les bandes passantes, les bandes interdites et les propriétés homogénéisées, dans la limite des grandes longueurs d'onde [6].

\section{HOMOGENEISATION D'UN MATERIAU POREUX.}

Le matériau étudié dans cette partie est le PVC, de masse volumique $\rho=1430 \mathrm{kgm}^{-3}$, dont les vitesses des ondes transversales et longitudinales sont respectivement $c_{\mathrm{T}}=972 \mathrm{~ms}^{-1}$ et $c_{\mathrm{L}}=2380 \mathrm{~ms}^{-1}$. Il comporte des pores cylindriques infiniment longs dans la direction $\mathrm{z}$. La distance entre deux pores voisins est constante et est prise égale à $2 \mathrm{~d}$ (Fig. 1). Les pores sont de section circulaire de diametre 2a. La porosité du matériau est définie comme étant le rapport de la surface des pores sur la surface totale. Une onde plane se

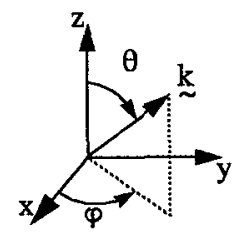
propage dans le milieu, caractérisée par son vecteur d'onde $\underset{\sim}{\mathbf{k}}$, repéré par les angles $\theta$ et $\varphi$. Une cellule est choisie arbitrairement d'épaisseur $h$ et est maillée par éléments finis. Les conditions de périodicité sont appliquées suivant les trois directions de l'espace. Les courbes de dispersion du matériau de porosité $P=0.385$ sont présentées à la figure 1 , pour les angles $\left(\theta=90^{\circ}, \varphi=90^{\circ}\right)$. Les trois branches issues de l'origine peuvent être assimilées à des droites lorsque $\mathbf{k}$ est petit, c'est-à-dire lorsque la longueur d'onde $\lambda$ est grande. Les pentes de ces droites sont les vitesses $\tilde{c}_{\mathrm{T} 1}, \tilde{c}_{\mathbf{T} 2}$ et $\tilde{c}_{\mathrm{L}}$ des ondes quasi-transversales et quasi-longitudinales dans le matériau.
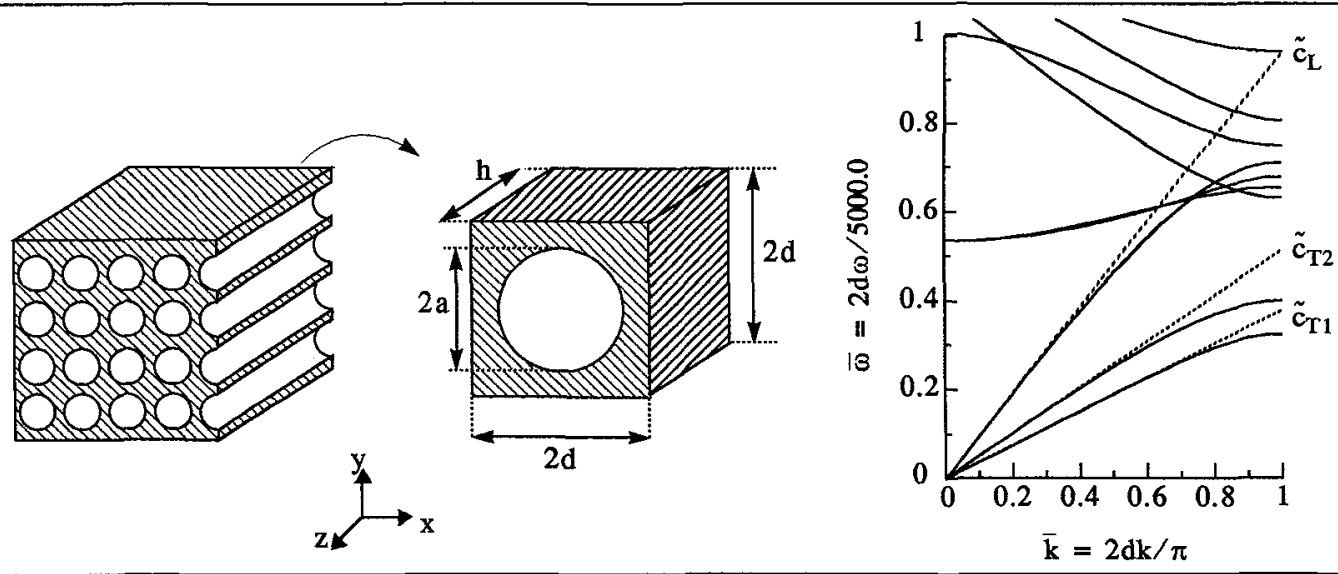

Figure 1 : Description du matériau périodique 2D, contenant des cavités cylindriques d'air. Courbes de dispersion pour $\mathrm{P}=0.385$, et $\theta=90^{\circ}, \varphi=90^{\circ}$. 
Une étude a montré que les vitesses $\tilde{c}_{\mathrm{L}}$ et $\tilde{\mathrm{c}}_{\mathrm{T}}$ sont dépendantes de la direction d'incidence du vecteur d'onde $\underset{\sim}{\mathrm{k}}[6]$. Ainsi, le matériau doit être homogénéisé sur un modèle anisotrope, dont le tenseur des constantes de rigidite est du type :

$$
[\tilde{c}]=\left[\begin{array}{cccccc}
\tilde{c}_{11} & \tilde{c}_{12} & \tilde{c}_{13} & 0 & 0 & 0 \\
\tilde{c}_{12} & \tilde{c}_{11} & \tilde{c}_{13} & 0 & 0 & 0 \\
\tilde{c}_{13} & \tilde{c}_{13} & \tilde{c}_{33} & 0 & 0 & 0 \\
0 & 0 & 0 & \tilde{c}_{44} & 0 & 0 \\
0 & 0 & 0 & 0 & \tilde{c}_{44} & 0 \\
0 & 0 & 0 & 0 & 0 & \tilde{c}_{66}
\end{array}\right]
$$

Afin de déterminer les constantes de rigidité effectives $\tilde{\mathbf{c}}_{11}, \tilde{\mathbf{c}}_{12}, \tilde{\mathbf{c}}_{13}, \tilde{\mathbf{c}}_{33}, \tilde{\mathbf{c}}_{44}$ et $\tilde{\mathbf{c}}_{66}$ du matériau poreux dans la limite des grandes longueurs d'onde, il faut calculer par la méthode des éléments finis les vitesses des ondes quasi-transversales et quasi-longitudinales pour deux directions différentes $\left(\theta=90^{\circ}, \varphi=45^{\circ}\right)$ et $\left(\theta=45^{\circ}, \varphi=0^{\circ}\right)$, puis résoudre un système d'équations données par les relations classiques de Christoffel [6]. A partir des coefficients homogenéisés, on peut déterminer, pour tous les angles $\theta$ et $\varphi$, les vitesses des ondes quasi-transversales $\tilde{\mathrm{c}}_{\mathrm{T} 1}(\theta, \varphi)$ et $\tilde{\mathrm{c}}_{\mathrm{T} 2}(\theta, \varphi)$ et quasi-longitudinales $\tilde{c}_{L}(\theta, \varphi)$. La figure 2 présente, en coordonnées polaires, les courbes de lenteur des ondes quasi-transversales et quasi-longitudinales, c'est-à-dire les variations des inverses des vitesses en fonction de l'angle d'incidence. Ces courbes, déterminées semi-analytiquement à partir des propriétés homogénéisées, sont tracées dans les plans $x y$ et $x z$ et sont comparées aux courbes obtenues par la méthode des éléments finis pour différentes directions de propagation.

La superposition parfaite des courbes montre que l'identification d'un matériau poreux périodique sur le modèle d'un matériau homogène anisotrope est exacte dans la limite des grandes longueurs d'onde.

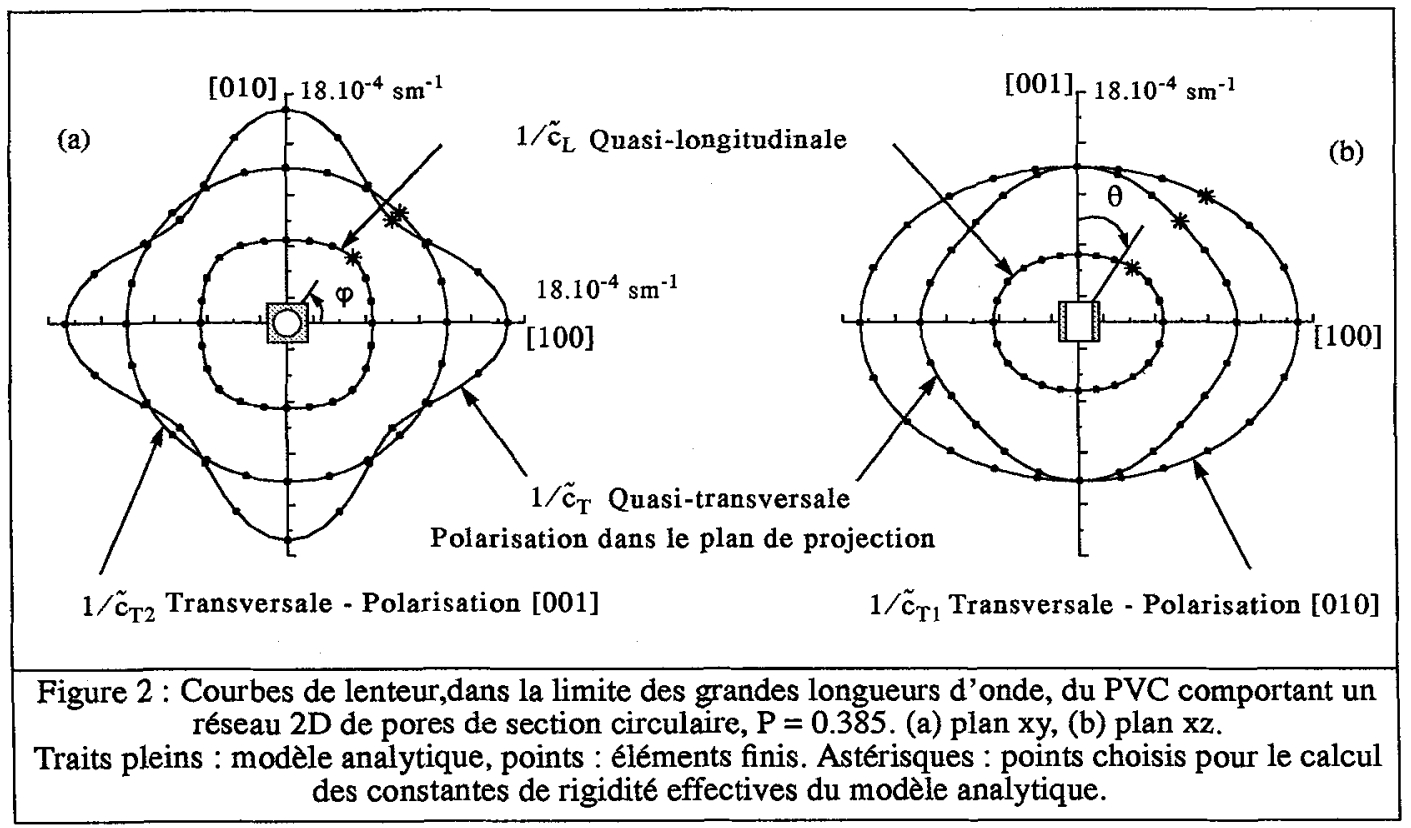




\section{ETUDE THEORIQUE ET EXPERIMENTALE DES MODES DE RESONANCE D'UNE PLAQUE PERFOREE PERIODIQUEMENT.}

Cette étude consiste à comparer pour deux plaques perforées périodiquement, les fréquences de résonance expérimentales à celles obtenues par la méthode des éléments finis, après homogénéisation du matériau sur le modèle anisotrope. Les plaques ( $309 \times 206 \times 20 \mathrm{~mm}$ ) sont en PVC et sont percées de 300 et de 600 trous de diamètre $7 \mathrm{~mm}$. Le tableau 1 donne, pour les trois premiers modes de résonance, la fréquence calculée par éléments finis $\mathrm{f}_{\mathrm{MEF}}$, la fréquence expérimentale $\mathrm{f}_{\mathrm{EXP}}$ et l'écart relatif des deux fréquences. Les fréquences des modes de résonance des plaques perforées ont chuté de façon notable par rapport aux fréquences de résonance de la plaque pleine. Les fréquences de résonance expérimentales sont en bon accord avec celles calculées par la méthode des éléments finis.

Tableau 1 : Fréquences théoriques et expérimentales pour les plaques de PVC.

\begin{tabular}{|c||c|c|c|c|c|c|c|c|c|}
\hline \multicolumn{1}{|c||}{} & \multicolumn{3}{c|}{ Plaque sans trou } & \multicolumn{3}{c|}{ Plaque 300 trous } & \multicolumn{3}{c|}{ Plaque 600 trous } \\
\hline $\mathrm{N}^{\circ}$ & $\begin{array}{c}\mathrm{f}_{\text {MEF }} \\
(\mathrm{Hz})\end{array}$ & $\begin{array}{c}\mathrm{f}_{\text {EXP }} \\
(\mathrm{Hz})\end{array}$ & $\begin{array}{c}\text { écart } \\
\%\end{array}$ & $\begin{array}{c}\mathrm{f}_{\text {MEF }} \\
(\mathrm{Hz})\end{array}$ & $\begin{array}{c}\mathrm{f}_{\text {EXP }} \\
(\mathrm{Hz})\end{array}$ & $\begin{array}{c}\text { écart } \\
\%\end{array}$ & $\begin{array}{c}\mathrm{f}_{\text {MEF }} \\
(\mathrm{Hz})\end{array}$ & $\begin{array}{c}\mathrm{f}_{\text {EXP }} \\
(\mathrm{Hz})\end{array}$ & $\begin{array}{c}\text { écart } \\
\%\end{array}$ \\
\hline \hline 1 & 305.6 & 307. & +0.5 & 282.2 & 272. & -3.6 & 192.2 & 180. & -6.3 \\
\hline 2 & 346.3 & 355. & +2.6 & 288.6 & 289. & +0.2 & 289.2 & 273. & -5.5 \\
\hline 3 & 702.8 & 701. & -0.3 & 634.1 & 621. & -2.1 & 480.5 & 459. & -4.5 \\
\hline
\end{tabular}

\section{CONCLUSION.}

Un modèle, reposant sur la méthode des éléments finis, a permis de décrire précisément le comportement des matériaux périodiques, sans restriction sur le champ de déplacement ou sur la direction d'incidence. L'exploitation des courbes de dispersion a permis de déterminer les propriétés homogénéisées de matériaux poreux sur le modèle de matériaux anisotropes, dans la limite des grandes longueurs d'onde. Cette homogénéisation a été validée expérimentalement par l'étude d'une plaque perforée périodiquement. Le premier de nos objectifs est d'étendre la procédure d'homogénéisation aux matériaux piézocomposites. Une extension de la méthode peut être menée pour les problèmes couplés fluide-solide et pour l'étude des ondes évanescentes dans les bandes interdites.

\section{Reférences.}

[1] Audoly C. et Duméry G., "Acoustic wave propagation in media containing two-dimensional periodically spaced elastic inclusions", Proc. Symp. on Physical Acoustics, Kortrijk Belgium juin 1990 (Ed. O. Leroy et M.A. Breazeale) pp 219-224.

[2] Achenbach J.D. et Kitahara M., "Harmonic waves in a solid with a periodic distribution of spherical cavities", J. Acoust. Soc. Am. 81 (1987) 595-598.

[3] Yamaguchi M., Hashimoto K.Y. et Makita H., "Finite element method analysis of dispersion characteristics for 1-3 type piezoelectric composites", IEEE Ultrason. Symp. (1987) 657-661.

[4] Langlet P., Hladky-Hennion A.-C. et Decarpigny J.-N., "Analyse de la propagation d'ondes acoustiques dans les structures périodiques à l'aide de la méthode des éléments finis", J. Phys., SuppI.III. n 4 C1 (1992) 1065-1068.

[5] Langlet P., “Analyse de la propagation des ondes acoustiques dans les matériaux périodiques à l'aide de la méthode des éléments finis", Thèse de Doctorat, Université de Valenciennes et du Hainaut-Cambrésis (1993).

[6] Dieulesaint E. et Royer D., Ondes élastiques dans les solides - Application au traitement du signal, (Ed. Masson, Paris, 1974). 